\title{
Broaden Views of the Impact of Lower Urinary Tract Symptoms on Healthcare
}

\author{
Ming-Ping $\mathrm{Wu}^{1,2 *}$ \\ ${ }^{1}$ Department of Obstetrics and Gynecology, Chi Mei Foundation Hospital, Taiwan \\ ${ }^{2}$ Department of Obstetrics and Gynecology, Fu-Jen Catholic University, Taiwan
}

*Corresponding author: Ming-Ping Wu, Department of Obstetrics and Gynecology, Chi Mei Foundation Hospital; 901, Chung Hwa Rd Yung Kang, Tainan, 710, Taiwan

Submission: 此 September 09, 2017; Published: 仵 October 11, 2017

Abbreviations: LUTS: Lower Urinary Tract Symptoms; IRRs: Incidence Rate Ratios; CI: Confidence Interval

\section{Short Communication}

Lower urinary tract symptoms (LUTS), which encompass storage, voiding, and postmicturition symptoms, are highly prevalent and recognized globally. Wu et. al [1] investigated the healthcare-seeking prevalence of those with lower LUTS based a nation-wide insurance data base in Taiwan. LUTS were associated with a low healthcare-seeking prevalence due to shame, embarrassment, social pressure, ora lack of knowledge of the problem. According to $\mathrm{Wu}$ et al. [1] the prevalence of healthcareseeking was only $2.31 \%$ in 2000 , with an increase to $3.24 \%$ in2005, and 3.84\% in 2009 [1]. There a rapid increase in LUTS healthcare-seeking prevalence may be due to both an increase in LUTS population, and the increase in the percentage of those with LUTS seeking medical assistance. However, the prevalence is still much lower than that in the real situation, e.g. $64.3 \%$ of the adult population had more than one LUTS by EPIC study in 2006 [2]. The low prevalence of healthcare-seeking may underestimate the impact of LUTS on health. Even though, the financial burden on the healthcare system is substantial. LUTS become more severe with age. Therefore, as the world population is aging, LUTS are becoming a substantial health-care burden as the number of aged people who want to maintain a good quality of life increases.

Later on $\mathrm{Wu}$ et al [1]. further reported that medical attendance for LUTS is associated with significant increases of subsequent risk of outpatient visits and hospitalizations with adjusted incidence rate ratios (IRRs) (95\% confidence interval (CI), 1.31 (1.291.32), and 1.48 (1.40-1.58), as compared with the control group respectively [3]. Although being an observational finding, it implies the significant impact of LUTS on the healthcare of different organs in addition to tradition urinary system. The increased risk of more healthcare-seeking and hospitalizations can be explained by a lower quality of life, lower threshold for seeking healthcare, and more concomitant co-morbidities, etc. Moreover, there may exist some other potentially hazardous precursors which predispose the development of major heath events [3].

Wu et al. [1] also observed an increased subsequent risk of hospitalization for acute cardiovascular events composite outcome LUTS group, with IRR1.34 (1.13-1.59), (mainly stroke, but not acute coronary syndrome) [4]. It is in concordant with that by Wehrberger et al. [4] in a longitudinal analysis with 10-year (mean 6.1 years) follow-up [5]. The explanations were possible due to some common risk factors for cardiovascular disease in patients with LUTS, e.g. multi factorial metabolic syndrome. LUTS patients may suffer from nocturia-induced sleep disturbances, non-dipping pattern of blood pressure changes, or adverse effects of medications for LUTS, which may result in autonomic nervous dysfunction, affective disorders, increased sympathetic activity, etc. A discrepant effect of LUTS on cardiovascular disease and stroke events can also come from the cognitive and cardiovascular effects of antimuscarinic agents, or a higher proportion of pre-existing syncope among patients with LUTS.

Furthermore, LUTS were also were identified as a risk factor of dementia 1.43 (1.26-1.61), LUTS might be a prodrome of cognitive deficiency or dementia [6]. Degeneration of global lobes, white matter lesions, and micro vascular brain disease has been associated with LUTS. LUTS might precede occurrence of cognitive disorder. Small vessel disease of the brain can cause incontinence, which may be the initial manifestation of dementia in some patients. Therefore, LUTS might be the early clinical symptom of the observed white matter changes and micro vascular brain disease.

In addition, patients with LUTS had a significantly higher prevalence of anxiety or depression than the matched controls (11.45\%vs.5.72\%), even after controlling socio demographic and other systemic variables, the odds ratios for anxiety, depression, either anxiety or depression, and both anxiety and depression, were 
$2.05,2.19,2.14$, and 2.56, respectively [7]. LUTS were associated with the stress-related common mental disorders, with an additive effect of anxiety and depression. It implies a psychological role in the pathogenesis or sequelae of LUTS. The studies by Wu et al. [1] and Wehrbergeret al. [4] broaden our current understanding of traditional organ-centric (urethra and bladder) LUTS concept. LUTS are better viewed as overlapping conditions involving multiple organ systems, rather than being focused on exclusively bladder and urethra.

\section{References}

1. Wu MP, Hsu YW, Weng SF, Ho CH, Wang JJ, et al. (2013) Healthcareseeking prevalence of lower urinary tract symptoms among National Health Insurance enrollees in Taiwan, 2000-2009. Urology 81(1): 61-65.

2. Irwin DE, Milsom I, Hunskaar S, Reilly K, Kopp Z, et al. (2006) Populationbased survey of urinary incontinence, overactive bladder, and other lower urinary tract symptoms in five countries: results of the EPIC study. Eur Urol 50(6): 1306-1314.
3. Wu MP, Weng SF, Hsu YW, Wang JJ, Kuo HC, et al. (2013) Medical attendance for lower urinary tract symptoms is associated with subsequent increased risk of outpatient visits and hospitalizations based on a nationwide population-based database. Plos One 8(3): e57825.

4. Wehrberger C, Temml C, Gutjahr G, Berger I, Rauchenwald M, et al. (2011) Is there an association between lower urinary tract symptoms and cardiovascular risk in men? A cross sectional and longitudinal analysis. Urology 78(5): 1063-1067.

5. Lin HJ, Weng SF, Yang CM, Wu MP (2013) Risk of hospitalization for acute cardiovascular events among subjects with lower urinary tract symptoms: A Nationwide Population-Based Study. Plos One 8(6): e66661.

6. Chiang $\mathrm{CH}, \mathrm{Wu} \mathrm{MP}$, Ho CH, Weng SF, Huang CC, et al. (2015) Lower urinary tract symptoms are associated with increased risk of dementia among the elderly: a nationwide study. Biomed Res Int 2015: p. 187819.

7. Lung-Cheng Huang C, Ho CH, Weng SF, Hsu YW, Wang JJ, et al. (2015) The association of healthcare seeking behavior for anxiety and depression among patients with lower urinary tract symptoms: a nationwide population-based study. Psychiat Res 226(1): 247-251. 